\title{
A venue-based HIV prevalence and behavioural study among men who have sex with men in Antwerp and Ghent, Flanders, Belgium, October 2009 to March 2010
}

W Vanden Berghe (Wvandenberghe@itg.be) ${ }^{1}$, C Nöstlinger ${ }^{1}$, A Buvé ${ }^{1}$, G Beelaert ${ }^{2}$, K Fransen ${ }^{2}$, M Laga ${ }^{1}$

1. Institute of Tropical Medicine HIV/AIDS Center (IHAC), Department of Public Health, Antwerp, Belgium

2. Institute of Tropical Medicine HIV/AIDS Center (IHAC), AIDS Reference Laboratory, Antwerp, Belgium

Citation style for this article:

Vanden Berghe W, Nöstlinger C, Buvé A, Beelaert G, Fransen K, Laga M. A venue-based HIV prevalence and behavioural study among men who have sex with men in Antwerp and Ghent, Flanders, Belgium, October 2009 to March 2010.

Euro Surveill. 2011;16(28):pii=19914. Available online: http://www.eurosurveillance.org/ViewArticle.aspx?Articleld=19914

This venue-based, cross-sectional study reports on human immunodeficiency virus (HIV) prevalence and behaviour of 649 men who have sex with men (MSM) in Antwerp and Ghent, Flanders, Belgium, from October 2009 to March 2010. Using time-location sampling, we found that HIV prevalence in MSM who attended different types of venue ranged from a high of $14.5 \%$ (95\% Cl: 8.9-20.1; $n=22$ in cruising venues to $4.9 \%$ (95\% Cl: 1.9-7.9; $\mathrm{n=10}$ ) in more general gay venues to $1.4 \%(95 \% \mathrm{Cl}: 0.0-3.6 ; n=3)$ at younger MSM venues. Of those who tested HIV positive $(n=35$, five were unaware of their HIV status or self-reported as being HIV negative. One in five respondents were of non-Belgian nationality. The results showed relatively high rates of testing for HIV (52.2\%; $95 \% \mathrm{Cl}: 47.8-56.2 ; n=288$ ) and other sexually transmitted infections (STIs) (57.4\%; 95\% Cl: $52.6-62.0 ; n=248)$ in the last 12 months. A majority of the men $(n=233)$ used condoms consistently during their last anal sexual contact with a casual partner; however, HIV-positive men who were aware of their serostatus $(n=30)$ reported less condom use with casual partners. This is the first such study in Belgium and the results constitute the evidence base for local, targeted interventions. Furthermore, our findings underscore the need for European crossborder cooperation to prevent HIV infection and other STIs among MSM.

\section{Introduction}

In most western countries, the number of diagnoses of new human immunodeficiency virus (HIV) infections in men who have sex with men (MSM) continues to rise [1]. In France, for example, it has been estimated that each year an additional $1 \%$ of MSM become infected with HIV - an increase described as detrimental to the collective health of future MSM communities [2].

In Belgium, 1,135 new HIV infections in the general population were reported in 2009 to the Belgian Federal Institute for Public Health [3], the highest number ever in a year. About $82 \%(n=244)$ of the new infections among men with Belgian nationality $(n=297)$ occurred in MSM. An analysis by age group revealed that younger MSM (aged 15-34 years) were disproportionally affected [3]. An increased uptake of HIV testing cannot fully explain this phenomenon [4] - several factors may contribute to increased rates of new HIV infections among MSM, including sexual risk taking [5] combined with a high prevalence of HIV infection in some networks of MSM [6].

In Belgium, as in many western countries, trends in HIV incidence are derived from registered diagnoses of HIV. However, such data have their limitations (as there is no additional background information for up to a third of registered new cases of HIV infection [3]) and may not reflect the real scope of the epidemic. In addition to HIV incidence estimates, population-based HIV prevalence estimates are needed to assess the burden of disease in MSM and to make realistic projections for health-service needs and prevention planning. More data are also needed on different types of MSM settings, as they are important for targeting prevention efforts.

The only HIV prevalence estimates for MSM in Belgium to date have relied on self-reported HIV status. In Flanders, 5.6\% $(n=1,736)$ of MSM in 2007 self-reported as HIV positive; among French-speaking residents of Brussels, 9\% $(n=942)$ of MSM in 2006 reported being HIV infected [7,8]. However, data for both studies were mainly collected through a variety of MSM websites and therefore do not give representative prevalence estimates. For Wallonia, no data are available.

In order to address several gaps in data for Flanders, in 2008 the Flemish Ministry of Wellbeing, Public Health and Family commissioned a population-based study on the prevalence of HIV infection and behaviour of MSM in Antwerp and Ghent, Flanders. We report here on the results of the study carried out between October 2009 and March 2010. 


\section{Methods}

Study design and estimated sample size

This study was carried out in the framework of 'Frequently Asked Questions' (FAQ), a series of behavioural and epidemiological research projects on HIV and other sexually transmitted infections (STIS) among MSM in Flanders [8]. The FAQ 2009 study design was a cross-sectional collection of blood samples and behavioural data, collected between October 2009 and March 2010. We set out to use time-location sampling to recruit men present at various venues in Antwerp and Ghent where MSM meet. This method has been shown to be successful in targeting hard-to-reach populations such as MSM [9].

Three main types or strata of MSM venues were defined on the basis of the age of the men who visited the venue and whether sexual contact was possible in the venue. The first stratum (cruising venues) comprised venues where sexual contact on site was possible, such as gay saunas, 'cruising' bars and sex clubs. The second (regular gay clubs/venues) consisted of more general MSM venues such as gay dance clubs or gay bars, where it is not possible to have sexual contact on site. The third (young MSM venues) consisted of settings where younger MSM meet, such as events organised by the regional organisation for lesbian, gay, bisexual and transgender youth, where sexual contact on site is also not permitted.

An inventory of all MSM venues in the Flemish cities of Antwerp and Ghent was then compiled from information obtained from the Internet and from community advisers, giving a total of 23 venues (10 cruising venues, nine regular gay bars/venues and four young MSM venues). Before the randomisation process, two types of enumeration were performed. Type I enumeration determined whether the venues gathered from the formative research were in fact venues that MSM attend. Next, type II enumeration was carried out to determine the number of eligible persons who attend a venue on a particular day and at a particular time period. On the basis of the enumeration data, 12 venues MSM venues (five cruising venues, five regular gay bars/venues and two young MSM venues) were randomly selected, without replacement, out of the list of 23, using STATISTICA v10. However, only seven owners or venue organisers agreed to collaborate: three cruising venues, two regular gay clubs/venues and two young MSM venues. The sample size for each stratum was calculated assuming a hypothetical prevalence of HIV infection of $15 \%$ for MSM at cruising venues and $5 \%$ in those at venues in the other two strata, as found in other studies [9-11]. In order to obtain a precision of $2.5 \%$, it was estimated that a total of 684 MSM would be required: 292 from cruising venues, 196 from regular gay clubs/venues and 196 from young MSM venues.

For the data collection, 12 volunteers were recruited from organisations involved in the prevention of HIV infection and other STIs and from community-based gay organisations. They received a half-day training on data collection procedures and ethical issues, to ensure optimal quality of data collection.

The volunteers were present at the venues on a Wednesday, Friday, Saturday or Sunday. Standardised time segments were used: three segments of three hours per location. Within the study period, time segments and days were randomised for data collection using STATISTICA $v$ 10. A team consisting of a principal investigator and up to three volunteers (depending on the size of the venue) visited each selected venue.

\section{Study population}

Respondents were recruited according to the following inclusion criteria: being male, aged 18 years or older and having had more than one same-sex sexual contact in the previous 12 months. Exclusion criteria were being physically or mentally unable to give informed consent and/or complete the questionnaire used to collect behavioural data (described below), having already participated in the study or showing signs (in speech and movement) of excessive drug or alcohol use.

\section{Procedures and data collection}

According to the principles of time-location sampling, the selection of MSM was random. The volunteers at the venue approached every other person entering or passing by the volunteers. The potential respondent was asked to participate: if they agreed, written informed consent was obtained. MSM who approached the volunteers were given some information on the research project and on HIV/STI prevention, but the volunteers explained that self-selection was not possible. During the training of the volunteers, the issue of selection bias had been discussed. Also, time-location sampling does limit selection bias, as there is multilevel randomisation.

Respondents were asked to complete a self-administered paper questionnaire available in Dutch and French. The questions $(n=32)$ were designed to gather data on socio-demographic characteristics, sexual orientation, partnership status, sexual contact according to partnership status, number of partners, condom use and position during anal sexual contact, sexual geography (i.e. places MSM frequent or strategies used to find partners), testing behaviour for HIV and other STIs, HIV status, history of other STIs and drug use. Questions on sexual activity were based on the United Nations General Assembly Special Session on HIV/AIDS (UNGASS) indicators [12]. The questions were pretested for clarity and feasibility among an MSM community test group during September 2009. Completing the questionnaire took the respondents about 10 minutes.

A different volunteer then collected a blood sample onto filter paper by means of a finger prick. As this method avoids using venous blood, it is therefore much less invasive - an important issue in the context 
of venue-based studies to encourage participation. A similar sampling procedure was used successfully in Montreal, Canada, and Paris, France $[10,13]$.

After each time segment of data collection, the samples and questionnaires were stored at the Institute of Tropical Medicine in Antwerp. All data were collected anonymously: a code linked a blood sample to the corresponding questionnaire. We checked for previous participation and the dataset was additionally checked for similarities in individual profiles (age and postal code) to exclude double entries.

The volunteers emphasised to the respondents that the HIV tests that would be carried out would not be used for diagnostic purposes. Respondents were given leaflets containing information on HIV testing and testing locations and an incentive was provided (a drink would be offered at the test, worth about three euros).

\section{Laboratory testing}

Blood samples were analysed at the AIDS Reference Laboratory of the Institute of Tropical Medicine, using Vironostika HIV Ag/Ab (bioMérieux), a fourth-generation test, and Enzygnost Anti HIV 1/2 Plus (Siemens), a third-generation test. If both tests were reactive, the sample was considered as HIV infected. Samples giving discordant results were considered as indeterminate and were not included in the analyses $(n=4)$.

\section{Statistical analysis}

Data were analysed with SPSS v 18 . Differences in proportions and means between different groups were tested for statistical significance using analysis of variance (ANOVA) and Tukey's post-hoc test.

\section{Ethical approval}

Ethical approval for the study was obtained from the Institutional Review Board of the Institute of Tropical Medicine, Antwerp, and the Ethics Committee of the University Teaching Hospital, Antwerp.

\section{Results}

\section{Socio-demographic characteristics}

of study participants

A total of 649 MSM participated in the study: 167 at cruising venues, 219 at regular gay clubs/venues and 263 at young MSM venues. Of the 649 questionnaires received, three were invalid; 582 of those who completed the questionnaire agreed to have their blood taken. Participation rates were calculated as the total number of respondents that participated divided by the total number of men approached by a recruiter, expressed as a percentage. The participation rate for completing the questionnaire was $58 \%$ $(n=167)$ at the cruising venues, $75 \%(n=219)$ at regular gay clubs/venues and $70 \%(n=263)$ at the young MSM venues. Because of the quantitative nature of the study, extra qualitative information on refusals was not obtained.
Mean age was highest among men recruited at the cruising venues ( 38.5 years) and, as expected, lowest in those recruited at the young MSM venues (26.9 years) (Table 1). At the cruising venues, $10.0 \%(n=17)$ of men were aged under 25 years; at the regular gay clubs/venues, this percentage was $25.0 \%(n=55)$ and at the young MSM venues, $40.0 \%(n=105)$.

Overall, $75.3 \%(n=489)$ of respondents were of Belgian nationality, $18.3 \%(n=119)$ were Dutch and $1.2 \%(n=8)$ were French nationals; the remaining men $(n=99)$ had different nationalities. About $64 \%(n=415)$ had $a$ degree. The vast majority of men $(94.1 \% ; n=611)$ were exclusively or primarily attracted to other men, while $2.6 \%(n=17)$ were equally attracted to men and women.

\section{Prevalence of HIV infection and undetected HIV infections}

At the cruising venues, $16.1 \%$ of men $(n=26)$ reported that they were HIV infected; the corresponding percentages for the regular gay clubs/venues and the young MSM venues were $5.8 \%(n=12)$ and $3.2 \%(n=8)$, respectively (Table 2). The proportion of men who did not know their HIV status was similar at the cruising venues and the regular gay clubs/venues: $16.1 \%(n=26)$ and $17.5 \%$ $(n=36)$, respectively. This was significantly lower than at the young MSM venues, where $26.0 \%$ of men $(n=64)$ reported they did not know their HIV status.

The prevalence of HIV infection was highest among the respondents at the cruising venues, $14.5 \%$ (22 of 152). It was $4.9 \%$ (10 of 205) among those at the regular gay clubs/venues and $1.4 \%$ (3 of 221) at the young MSM venues (Table 2). The differences between strata were significant. Overall, $14.3 \%(n=5)$ of HIV-positive MSM thought they were HIV negative or were unaware of their status. This proportion was different in the different strata: at the cruising venues, $5.0 \%$ of men $(n=1)$ were unaware of their HIV-positive status; at the regular gay clubs/venues, this percentage was $30.0 \%(n=3)$ and at the young MSM venues, $25.0 \%(n=1)$. These differences were not statistically significant, but the numbers were small. Five HIV-negative men self-reported being HIV positive. Four other respondents had discordant results between the two HIV tests.

Table 2 also presents HIV prevalence by age group in the different types of venue. Prevalence was highest among men at the cruising venues, in all age groups. The prevalence in men aged 25 years or younger and in men 40 years or older was similar in regular gay clubs/ venues and in young MSM venues.

\section{Testing for HIV infection and other STIs}

Overall, $88.0 \%(n=531)$ had ever been tested for HIV and about half had been tested in the previous 12 months. Men at young MSM venues were least likely to have ever been tested $(81.4 \% ; n=184)$, while the percentage of men ever tested was similar at the cruising venues $(92.6 \% ; n=150)$ and the regular gay clubs/venues (90.2\%; $n=194)$ (Table 1 ). 

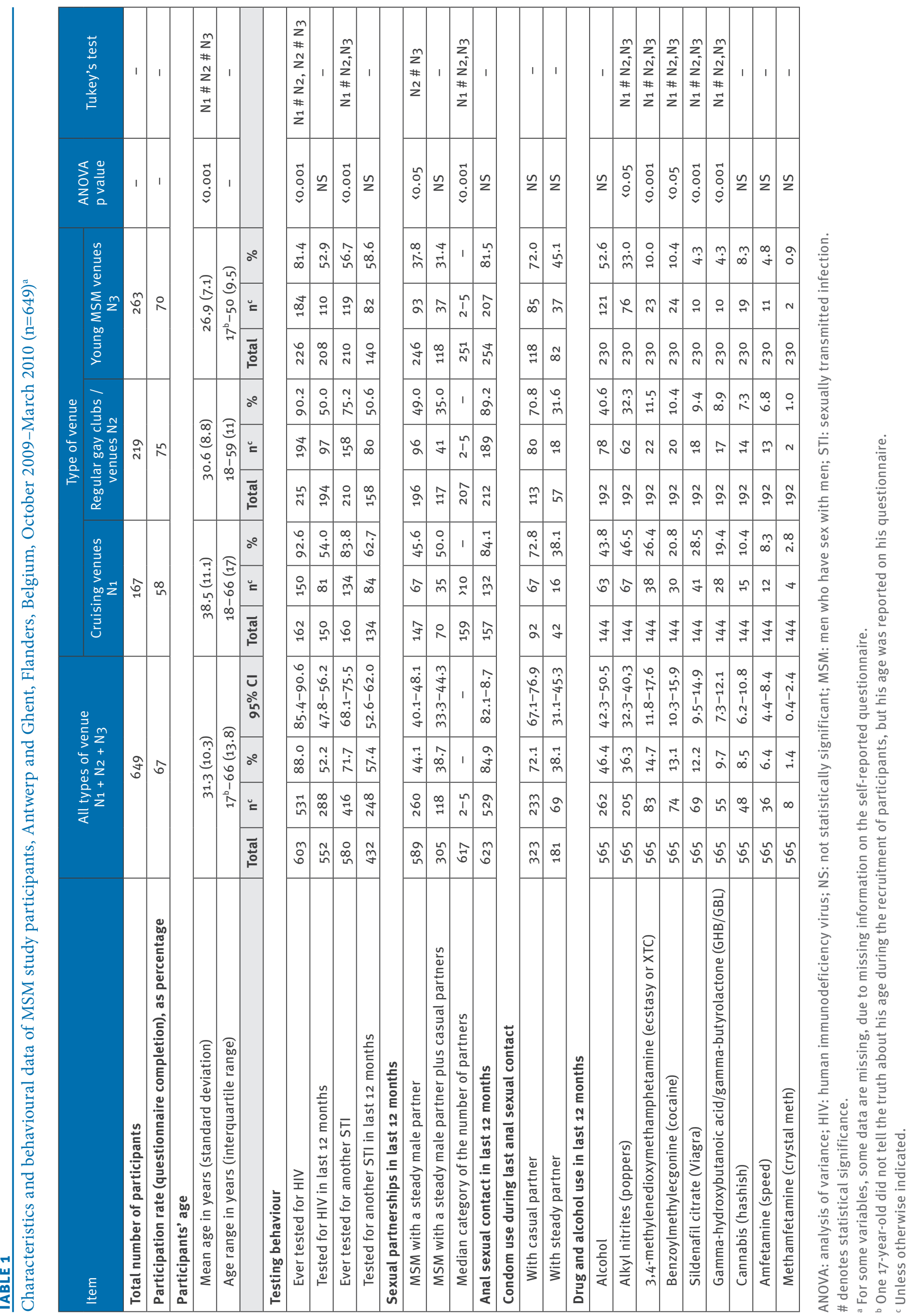

Testing rates ever for STIs were also high: $83.8 \%$ $(n=134)$ for respondents at cruising venues, which was significantly higher than among those at regular gay clubs/venues $(75.2 \% ; n=158)$ and young MSM venues (56.7\%; $n=119)$. Overall, $57.4 \%(n=248)$ of respondents had been tested for STIs other than HIV in the previous 12 months (Table 1). Of the respondents who had been tested for other STIs, about $16.5 \%(n=41)$ had been diagnosed with one or more STIs; for men recruited at cruising venues, this was $28.6 \%(n=24)$.

The most frequently reported STIs among those tested were gonorrhoea (10.1\%; $n=29)$, chlamydial infection $(9.1 \% ; n=26)$ and syphilis ( $8 \% ; n=23)$. There were 13 cases of hepatitis B (4.5\%) and eight cases of hepatitis C (2.8\%). Significantly more syphilis cases were reported in respondents in cruising venues $(15.4 \%$, Tukey's test p<0.05) compared with the other two strata ( $4.8 \%$ in regular gay clubs/venues and $5.8 \%$ in young MSM venues, $n=6)$. Chlamydial infection was significantly less likely to be reported by respondents from young MSM venues (in 3.9\% $(n=4)$ of respondents, whereas the percentage was $9.5 \%(n=10)$ in regular gay clubs/venues and $15.4 \%(n=12)$ in cruising venues; Tukey's test p<0.05). During the previous 12 months, $24.0 \%(n=10)$ of the respondents diagnosed with an STI other than HIV were diagnosed with at least one other STI.

\section{Sexual partnerships and sexual behaviour}

A total of $260(44.1 \%)$ of the respondents reported having had a steady male partner in the previous 12 months. For those in the younger MSM venues, this was $37.8 \%(n=93)$ (Table 1$)$. Of all respondents who reported a steady relationship, about one third $(n=118)$ had also had casual sexual partners in the previous 12 months; for respondents at cruising venues, this was $50.0 \%(n=35)$. The median category of number of sexual partners in the previous 12 months was two to five for respondents at regular gay clubs/venues and young MSM venues, which was significantly lower than that for respondents in cruising venues, where the median category was more than 10.

Overall, $84.9 \%(n=529)$ of the respondents reported having had anal sexual contact during the previous 12 months. One third acted almost exclusively as the receptive partner, slightly more reported insertive anal sexual contact and about one third reported both. A total of $61.9 \%(n=112)$ of the respondents had not used a condom with their steady partner at the last sexual encounter. There were no differences by respondent's HIV status in condom use during the last anal sexual contact. However, when having anal sexual contact with a casual partner or a sex-buddy (a sexual partner who was known to the person, but was not their steady partner), HIV status seemed to affect condom usage: among HIV-negative respondents $(n=552), 412(74.6 \%)$ consistently used a condom during their last anal sexual contact with casual partners, compared with $44.1 \%$ $(n=15)$ among the HIV-positive respondents.
There were no significant differences between respondents by type of venue in the self-reported strategies that were used to search for sexual partners. Our results indicate that few MSM look for sexual partners in only one single type of venue or use only one strategy exclusively: respondents in all venue types found sexual partners through the Internet, via friends or at regular gay clubs or bars, although respondents in the young MSM venues were significantly more numerous at regular gay clubs or bars and had used the Internet more frequently to find sexual partners during the last 12 months. Overall, $76.6 \%$ of all respondents $(n=464)$ reported having had sexual contact at their home after finding a sex partner through any strategy or at any of the venues. As expected, respondents from cruising venues were less likely to have sexual contact at home after finding a casual sex partner though this difference was not statistically significant.

\section{Drug and alcohol use}

The respondents reported using a range of different drugs in the previous 12 months, just before or during sexual contact (Table 1). The drugs included alkyl nitrites (poppers), 3,4-methylenedioxymethamphetamine (ecstasy or XTC), cannabis (hashish) and amfetamine (speed). Combinations of all of the above drugs were also reported. Significantly higher rates of use of alkyl nitrites (poppers), sildenafil citrate (Viagra), 3,4-methylenedioxymethamphetamine (ecstasy or XTC), gamma-hydroxybutanoic acid/gamma-butyrolactone $(\mathrm{GHB} / \mathrm{GBL}$ ) and benzoylmethylecgonine (cocaine) use were reported by respondents in cruising venues compared with those in the other venue types.

Excessive alcohol use was also reported. Respondents at young MSM venues used significantly more alcohol than those at regular gay clubs/venues.

\section{Discussion}

This is the first study in Belgium to estimate HIV prevalence among MSM visiting different types of venue. The lack of such research in the past may be due to the overall lack of knowledge of MSM populations and to difficulties in reaching these men for population-based research. The MSM sexual subculture is extremely diverse and caters for specific sexual desires. HIV prevalence research tends to focus primarily on cruising venues, where sexual contact on the premises is possible. By analysing three different types of venue, we were able to differentiate between specific venue types and our findings thus contribute to developing targeted prevention strategies.

Recruiting MSM from different settings and taking blood samples through a finger prick seemed feasible and was generally well accepted. However, a limitation of our study was that five of the 12 owners of the venues that were initially approached declined to participate in the study. They rarely gave a meaningful explanation for not participating. Further research could shed light on the venue owners' motivation for refusal. The 
exclusion of these venues limited the application of the time-location sampling framework and the representativeness of the data for the MSM scene in Flanders. In addition, some of the men did not want to provide a blood sample as they said they feared the pain caused by a finger prick. Given the setting in which the samples were collected, this could have been said to hide the other reasons for refusing to be tested; however, this could not be explored in more detail in our study. Further, self-completion of the questionnaire in these venues resulted in some questions not being answered. Nevertheless, use of principles of the time-location sampling methodology means that our results should be representative for MSM present at the venues that were visited in the cities of Antwerp and Ghent, but the results cannot be generalised to the overall population of MSM in Flanders or the whole of Belgium. It is noteworthy, however, that analysis of the respondents' place of residence (by postal code) showed that the Belgian MSM in the study came from all over the country.

Our study found that the prevalence of HIV infection in men in the MSM venues that we analysed ranged from $14.5 \%$ in cruising venues, to $4.9 \%$ in regular gay clubs/ venues, to $1.4 \%$ at young MSM venues. These differences in prevalence can be partly explained by differences in age. As the age of MSM at cruising venues was on average higher and HIV infection is more prevalent in older age groups, it is not surprising to find more HIV infections within these settings. However, in MSM aged 25 years and under who were present at cruising venues, the prevalence was $10 \%$. These young men frequented a greater variety of settings and used diverse strategies to find sexual partners, while they reported less frequent HIV testing.

Our prevalence data are in line with other European venue-based research among MSM, which found a range of prevalence estimates. A study in the United Kingdom in 2007 found the prevalence of HIV infection in MSM to be $9.0 \%$ in Manchester, $12.0 \%$ in London, and $14.0 \%$ in Brighton, and about a third of infections were unknown by the respondents [14]. In Switzerland, the estimated prevalence of HIV infection among MSM in Geneva in 2005 was $11 \%$ [15]. More recently, in Barcelona, Spain, the figure was $17.0 \%$; in Verona, Italy, $11.8 \%$; in Bratislava, Slovakia, $6.1 \%$; in Bucharest, Romania, 4.6\%; in Ljubljana, Slovenia, $5.1 \%$; and in Prague, Czech Republic, $2.6 \%$. Among MSM aged under 25 years, the prevalence was $4.9 \%$ in Verona, Italy, and $12.5 \%$ in Barcelona, Spain, in 2008 to 2009 [9]. The most recent European study, among MSM in Paris, France, in 2009 found a prevalence of $17.7 \%$, of whom $19.7 \%$ were unaware of their status [10]. However, caution has to be exercised when comparing these results as the data were collected in different urban contexts and time periods, using different methodologies. In our study, the percentage of men unaware of their HIV-positive status was lower across all settings (14.3\%), although it was relatively high in the regular gay clubs/venues (30.0\%) and young MSM venues $(25.0 \%)$. This finding could be explained by a lower uptake of HIV testing by younger MSM.

We found relatively similar but high rates of testing for HIV during the last 12 months, compared with results from a similar study in 2009 in France, a neighbouring country (where the rate of HIV testing was 63.0\%) [10]. Respondents in the cruising venues in our study had been tested significantly more frequently for both HIV and other STIs than those from the other venue types. The higher uptake of lifetime testing in MSM in cruising venues corresponds to the higher risk of exposure within the settings they frequent. Further, the MSM present at cruising venues are generally older and may therefore have known about or experienced HIV screening for a longer time.

A majority of the men in our study reported condom use consistently during the last anal sexual contact with a casual partner, across all strata. However, HIV-positive men who were aware of their serostatus reported less condom use with casual partners and/or sex-buddies. Further, prevalence of HIV infection was highest among the participants from cruising venues - where sex in public is possible on site - even after stratifying by age group. Although it may appear that a certain group of HIV-positive MSM take no preventive measures at all, this may not always be the case. Research has shown that it is within the sexual networks of MSM that harm reduction strategies such as strategic positioning (HIV-positive men assume the receptive or 'passive' position, while HIV-negative men take the insertive or 'active' position) and serosorting (HIV-positive men have sex only with other HIV-positive men, while HIVnegative men only do so with other HIV-negative men) are widely practised and accepted as forms of preventive behaviour [6]. Within epidemiological research this process of seroadaptation has often been ignored [16]. However, as a substantial number of HIV-positive respondents in our study believed themselves to be HIV negative, these strategies cannot be considered as reliable.

The use of certain drugs was higher among participants in the cruising venues. Drug use and sexual risk behaviour among MSM attending these sex venues were reported to be high, as was reported in a study in 2009 on highly sexually active MSM attending cruising venues and parties in New York, United States [17]. From a public health perspective, prevention strategies for HIV/STIs and drug consumption are generally difficult to control in MSM venues, as they are privately owned. The relationship between drug taking, mental health and sexual risk behaviour is pivotal in understanding the HIV epidemic among MSM, not only among men attending these venues but for the whole community [18]. The use of poppers and Viagra has been shown to be associated with recent seroconversion [19] and sexual risk behaviour [20-23]. While the purpose of using these drugs is the enhancement of sexual experience [23], interventions tackling multiple drug use need to be 
part of prevention strategies for the specific high-risk subgroup of users, including HIV-positive men [24].

With regard to venues frequented or strategies used by MSM to seek sexual partners, there appear to be differences according to age group. For instance, data from the Netherlands suggest that younger MSM seek out sexual contact more frequently through the Internet and friends, and at regular gay bars or parties, whereas older MSM prefer saunas, public places (e.g. public parks and car parks), 'darkrooms' (darkened rooms, sometimes located in a cruising bar, gay sauna or other place where sexual activity is possible), sex cinemas and sex clubs [25]. In our study, the role of the Internet appeared to be important. A majority of the respondents sought sexual partners on the Internet and then met in their homes. Therefore it is imperative that new interventions focus on providing information on HIV infection and other STIs on the Internet and work through digital interaction on changing sexual risk behaviour.

The complexity of the psychological, biological and social-structural elements that define the HIV epidemic among MSM requires a combination prevention solution. Our data show clearly that targeting only one element is not enough [6]. Our results constitute the evidence base for local targeted prevention, for policy changes directed at these specific settings and they form a baseline for analysing trends in HIV prevalence to inform prevention planning and monitor progress. We know from residence analysis that one in five men participating in the study came from neighbouring countries. Further, as the epidemiology of HIV infection among Belgian MSM mirrors developments in most western countries, there is a need for cross-border cooperation on research and development of interventions and policies [26]. Within such a framework, we could move towards a European-wide HIV prevention plan for MSM, as has been suggested [27].

\section{References}

1. European Centre for Disease Prevention and Control (ECDC)/ World Health Organization Regional Office for Europe. HIV/ AIDS surveillance in Europe 2009. Stockholm: ECDC; 2010.

2. Le Vu S, Le Strat Y, Barin F, Pillonel J, Cazein F, Bousquet V, et al. Population-based HIV-1 incidence in France, 2003-08: a modelling analysis. Lancet Infect. Dis. 2010;10(10):682-7.

3. Sasse A, Verbrugge R, Buziarist J, Van Beckhoven D. Epidemiologie van AIDS en HIV infectie in België (toestand op 31 december 2009) [Epidemiology of AIDS and HIV infection (31 December 2009]. Brussels: Scientific Institute of Public Health, Public health and Surveillance; 2010. Dutch.

4. Sullivan PS, Hamouda O, Delpech V, Geduld JE, Prejean J, Semaille C, et al. Reemergence of the HIV Epidemic among men who have sex with men in North America, western Europe, and Australia, 1996-2005. Ann Epidemiol. 2009;19(6):423-31.

5. Folch C, Casabona J, Munoz R, Gonzalez V, Zaragoza K. [Increase in the prevalence of HIV and in associated risk behaviors in men who have sex with men: 12 years of behavioral surveillance surveys in Catalonia (Spain)]. Gac Sanit. 2010;24(1):40-6. Spanish.

6. Hart GJ, Elford J. Sexual risk behaviour of men who have sex with men: emerging patterns and new challenges. Curr Opin Infect Dis. 2010;23(1):39-44.
7. Martens V, Huynen P. Knowledge and behaviour of men who have sex with men towards HIV/AIDS in the French Community of Belgium. Arch Public Health. 2006;64:13-26.

8. Defraye A, Sasse A. SOA-surveillance in AIDS Referentiecentra in België - Jaarrapport 2008 [STI surveillance in Belgian AIDS reference centers - yearly report 2008]. Brussels: Scientific Institute of Public Health; September 2009. Dutch.

9. Mirandola M, Folch Toda C, Krampac I, Nita I, Stanekova D, Stehlikova D, et al. HIV bio-behavioural survey among men who have sex with men in Barcelona, Bratislava, Bucharest, Ljubljana, Prague and Verona, 2008-2009. Euro Surveill. 2009;14(48):pii=19427. Available from: http://www. eurosurveillance.org/ViewArticle.aspx?Articleld=19427

10. Velter A, Barin F, Bouyssou A, Guinard J, Le Vu S, Pillonel J, et al. High prevalence and incidence of HIV infection among men who have sex with men recruited in commercial gay venues in Paris (France): Prevagay 2009 survey. Poster presentation at AIDS 2010, 18-23 July 2010, Vienna, Austria. Available from: http://www.sneg.org/documents/PREVAGAY/AIDS_PREVAGAY_ VELTER_2.pdf

11. Sifakis F, Hylton JB, Flynn C, Solomon L, MacKellar DA, Valleroy LA, et al. Prevalence of HIV infection and prior HIV testing among young men who have sex with men. The Baltimore young men's survey. AIDS Behav. 2010;14(4):904-12.

12. Joint United Nations Programme on HIV/AIDS (UNAIDS). Monitoring the Declaration of Commitment on HIV/AIDS guidelines on construction of core indicators: 2008 reporting. Geneva: UNAIDS; 2007. UNAIDS/07.12E / JC1318E.

13. Lambert G, Cox J, Tremblay F, Tremblay C, Alary M, Lavoie, et al. Recent HIV testing behaviour among men who have sex with men (MSM) in Montreal: results from the ARGUS 2005 survey. Can J infect Dis Med Microbiol. 2006;17(suppl A):45A.

14. Dodds JP, Johnson AM, Parry JV, Mercey DE. A tale of three cities: persisting high HIV prevalence, risk behaviour and undiagnosed infection in community samples of men who have sex with men. Sex Transm Infect. 2007;83(5):392-6.

15. Häusermann M, Wang J. Projet santé gaie.Les premiers résultats de l'enquête sur la santé des hommes gais de Genève [Gay health project. The first results from the health survey among gay men in Geneva.. Geneva: Dialogai; 2003. French.

16. McConnell JJ, Bragg L, Shiboski S, Grant RM. Sexual seroadaptation: lessons for prevention and sex research from a cohort of HIV-positive men who have sex with men. PLoS One. 2010;5(1) ):e8831.

17. McNeely J, Silvera R, Torres K, Bernstein K, Aberg J, Gourevitch $M$, et al. Current substance misuse and HIV risk behavior among highly sexually active men who have sex with men (MSM) attending commercial sex venues, events and parties (CSVEP) in New York City. J Gen Intern Med. 2010;25:250.

18. Safren SA, Reisner SL, Herrick A, Mimiaga MJ, Stall RD. Mental health and HIV risk in men who have sex with men. J Acquir Immune Defic Syndr. 2010 Dec 15;55 Suppl 2:S74-7.

19. Carey JW, Mejia R, Bingham T, Ciesielski C, Gelaude D, Herbst $\mathrm{JH}$, et al. Drug use, high-risk sex behaviors, and increased risk for recent HIV infection among men who have sex with men in Chicago and Los Angeles. AIDS Behav. 2009;13(6):1084-96.

20. Kim AA, Kent CK, Klausner JD. Increased risk of HIV and sexually transmitted disease transmission among gay or bisexual men who use Viagra, San Francisco 2000-2001. AIDS. 2002;16(10):1425-8.

21. Nettles CD, Benotsch EG, Uban KA. Sexual risk behaviors among men who have sex with men using erectile dysfunction medications. AIDS Patient Care STDS. 2009;23(12):1017-23.

22. Spindler HH, Scheer S, Chen SY, Klausner JD, Katz MH, Valleroy LA, et al. Viagra, methamphetamine, and HIV risk: results from a probability sample of MSM. Sex Transm Dis. 2007;34(8):586-91.

23. Prestage G, Grierson J, Bradley J, Hurley M, Hudson J. The role of drugs during group sex among gay men in Australia. Sex Health. 2009;6(4):310-7.

24. Mayer KH, O’Cleirigh C, Skeer M, Covahey C, Leidolf E, Vanderwarker R, et al. Which HIV-infected men who have sex with men in care are engaging in risky sex and acquiring sexually transmitted infections: findings from a Boston community health centre. Sex Transm Infect. 2010;86(1):66-70.

25. Hospers HJ, Roos E, Zuilhof W. Schorer monitor 2009. Amsterdam: Universiteit Maastricht - Schorer; 2009.

26. 26. Beyrer C. Global prevention of HIV infection for neglected populations: men who have sex with men. Clin Infect Dis. 2010;50 Suppl 3:S108-13.

27. Likatavicius G, Klavs I, Devaux I, Alix J, Nardone A. An increase in newly diagnosed HIV cases reported among men who have sex with men in Europe, 2000-6: implications for a European public health strategy. Sex Transm Infect. 2008;84(6):499-505. 